\title{
Stochastic Control of Metabolic Pathways
}

\author{
Andrea Rocco \\ Department of Mathematical Sciences, University of Bath \\ BA2 7AY Bath, United Kingdom
}

(Dated: October 25, 2018)

\begin{abstract}
We study the effect of extrinsic noise in metabolic networks. We introduce external random fluctuations at the kinetic level, and show how these lead to a stochastic generalization of standard Metabolic Control Analysis. While Summation and Connectivity Theorems hold true in presence of extrinsic noise, control coefficients incorporate its effect through an explicit dependency on the noise intensity. New elasticities and response coefficients are also defined. Accordingly, the concept of control by noise is introduced as a way of tuning the systemic behaviour of metabolisms. We argue that this framework holds for intrinsic noise too, when time-scale separation is present in the system.
\end{abstract}

\section{INTRODUCTION}

Stochastic fluctuations represent an important contribution to complex behaviours of biological systems. Stochasticity appears as a fundamental dynamical mechanism, which does not only generate phenotypic diversity [6], but also plays a major role at many different levels. Selection of alternative pathways in epigenetic switches [4], or synchronisation of multicellular systems [33], are just two of the many processes which not only are influenced by stochasticity, but seem to use it to perform in optimal way [32].

In molecular biology two classes of stochastic fluctuations are particularly relevant. The first class is related to the low copy number of chemical species. In particular, if $N$ is the number of molecules in the system, fluctuations in $N$ lead to an associated statistical noise with intensity of the order of $N^{-1 / 2}$. While continuous deterministic descriptions in terms of average concentrations can effectively capture the relevant dynamics for $N$ large, when $N$ is small fluctuations may become huge, and noise cannot be neglected [9]. Gene regulation is a typical example, as it may be affected by large fluctuations due to the low copy number of transcription factors [5]. Fluctuations associated with the intrinsic discreteness of the collisional processes among single molecules are usually referred to as intrinsic (or internal) noise.

On the other hand, the behaviour of a biochemical system depends also on a number of control parameters. Some of these relate directly to the macroscopic environment, such as for instance illumination conditions, or pH levels. Others have a more microscopic origin, but still exhert a control on the sytem which is independent of its intrinsic dynamics. In gene regulatory networks, for instance, factors acting globally on all genes, such as abundance of RNA polymerase, can change the global efficiency of transcription factors, and ultimately contribute to tuning gene expression levels 26]. Similarly, reaction constants, enzyme activities, or input signals critically control the functional behaviour of metabolic pathways. In thise sense, all these parameters are external to the system. Fluctuations of external parameters define a second type of noise, usually referred to as extrinsic, or external.

The effect of extrinsic noise can be highly non-trivial and counterintuitive. Extrinsic noise in homogeneous chemical systems has been shown to provoke noise-induced transitions [22], and recently it has been identified as a mechanism for creating and sustaining spatio-temporal patterns in spatially extended systems [11]. In gene networks the interplay between intrinsic and extrinsic noise has been recently analysed in [25, 28, 29].

In this paper we plan to extend these findings to metabolic networks. We shall focus on the case when the copy number of molecules is large enough for intrinsic noise to be negligible, so that a continuous description in terms of average concentrations is feasible, but at the same time the system experiences extrinsic stochastic fluctuations. Deterministic rate equations become thus stochastic differential equations.

Given the description in terms of rate equations, it is ideally desirable to define other, more "systemic" approaches, which explain how global properties of the pathway, such as fluxes and concentrations, depend on local variables, such as enzyme activities. To this aim, in the specific case of metabolic networks a useful strategy -the so-called Metabolic Control Analysis (MCA) [15]- has been developed, and has become nowadays a popular quantitative framework for investigating control and regulation of metabolisms.

Mathematically MCA is a sensitivity analysis, and is based on a proper manipulation of the rate equations. It consists in perturbing the parameters of the system, and evaluating the corresponding change in steady state fluxes and concentrations. Perturbations need to be small so that a linear approximation can hold, even if in principle the analysis can be carried on including higher order terms. The effect of the perturbation is represented by a control coefficient, which expresses the control that a certain rate exerts on the steady state variable of interest. 
A fundamental property of control coefficients is their interdependence. This is formalized in the so-called Summation Theorems [15] for both flux and concentration control coefficients: For any flux, the sum of the flux control coefficients of all the rates in the pathway equals one. Similarly, for any metabolite, the sum of the concentration control coefficients of all the rates in the pathway equals zero. These properties demonstrate the actual complexity of the system. In fact, control on systemic variables is typically distributed among all the enzymes in the system, being the respective control coefficients often of the same order of magnitude and constrained by the Summation Theorems. This implies that none of them alone is capable of determining the systemic properties of the network [3].

Control coefficients are also responsible for mediating the way local perturbations climb up to the systemic level. This is the content of the so-called Connectivity Theorems and Partitioned Response relations. The response of the system is the sum of the product of the local sensitivity of the individual rates to perturbations (elasticities) times the control that those rates exert on the system variable of interest. Connectivity Theorems and Partitioned Response, together with the Summation Theorems, define the mathematical structure of Metabolic Control Analysis.

We present here a novel framework, which allows the description of extrinsic fluctuations within Metabolic Control Analysis. Our approach shows that when considering extrinsic stochasticity, Summation Theorems are still valid in the standard form, but with control coefficients explicitly dependent on the noise intensity. The same is true for Connectivity Theorems and Partitioned Response, and for elasticities and response coefficients. Our findings suggest a reinterpretation of noise as an "active" player in the global dynamics of the network, rather than as a mere uncontrollable variable. On the one hand, noise may allow the metabolic pathway to access dynamical regions which would be otherwise out of reach if the dynamics were purely deterministic. On the other hand, it may provide us with an alternative mechanism to exert external control on the regulation of metabolisms.

In Section 2 we define the stochastic rate equations associated with extrinsic noise, and we illustrate how the corresponding stationary probability distribution shows non-trivial modifications with respect to the deterministic case. Section 3 is the core of the paper. When extrinsic stochasticity is implemented, the general principles of MCA still hold, and incorporate in a natural way the effect of noise. An explicit illustration of our approach is presented in Section 4, and we finally conclude in Section 5.

\section{KINETICS WITH EXTRINSIC STOCHASTICITY}

Consider a metabolic network with $N$ metabolites and $M$ reactions. In the homogeneous (well-stirred) situation, spatial dependencies are negligible, and the network is dynamically represented in terms of the following set of ordinary differential equations:

$$
\frac{d \mathbf{c}}{d t}=\mathbf{f}(\mathbf{c}, \boldsymbol{\mu}),
$$

with

$$
f_{i}=\sum_{j=1}^{M} S_{i j} v_{j}, \quad i=1, \ldots, N .
$$

The $N \times M$ matrix $S_{i j}$ is the so-called stoichiometry matrix, and $v_{j}=v_{j}(\mathbf{c}, \boldsymbol{\mu})$ are reaction rates. Here $\mathbf{c}=\left\{c_{1}, \ldots, c_{N}\right\}$ represents the vector of concentrations, and $\boldsymbol{\mu}=\left\{\mu_{1}, \ldots, \mu_{R}\right\}$ is a vector of $R$ parameters, such as reaction constants, enzyme concentrations, illumination conditions, input signals, etc.

We are interested in the situation when these parameters behave dynamically, and in particular exhibit random fluctuations. Experiments can be designed on purpose to investigate the effect of extrinsic fluctuations. For instance, in [18] the effect of stochastic fluctuations on the substrate injection rate in a model for glycolytic oscillations is analyzed, and a new noise-dependent dynamical regime of oscillations is identified. On the other hand, extrinsic noise can originate from intrinsic noise after some reduction procedure has been carried out. For instance the derivation of the Michaelis-Menten kinetics relies on the assumption of rapid equilibration of the enzyme [15, 20]. As a consequence, the dependency on the enzyme concentration is parametric in the Michaelis-Menten form, and intrinsic fluctuations on enzyme activity are then perceived as extrinsic in the reduced model. Enzyme activity can fluctuate because of the stochastic nature of gene expression [24], or due to protein conformational changes [10].

Hence, we model extrinsic random fluctuations on the parameters $\boldsymbol{\mu}$ by setting for $i=1, \ldots, R$ :

$$
\mu_{i} \rightarrow \mu_{i}(t)=\bar{\mu}_{i}+\xi_{i}(t),
$$


where $\bar{\mu}_{i}$ is the average value of $\mu_{i}$, and the stochastic process $\xi_{i}(t)$ satisfies the equation

$$
\frac{d \xi_{i}}{d t}=-\frac{\xi_{i}}{\tau_{i}}+\frac{\varepsilon_{i}^{1 / 2}}{\tau_{i}} \zeta_{i}(t)
$$

Eq. (44) defines the well-known colored Ornstein-Uhlenbeck noise [12]. The parameters $\tau_{i}$ play the role of the inverse correlation time of the noise, while the parameters $\varepsilon_{i}$ are the noise intensities. These account for different parameters to respond with different sensitivities to the external fluctuations. The case when one or more parameters do not fluctuate at all, is selected by choosing the appropriate $\varepsilon_{i}$ 's equal to zero. Furthermore, the assumption that

$$
\varepsilon_{i}\left(\mu_{i}\right) \rightarrow 0 \quad \text { as } \quad \mu_{i} \rightarrow 0 \text { for } i=1, \ldots, R
$$

guarantees that $\mu_{i}$ stays positive, and centered around $\bar{\mu}_{i}$. Finally the noises $\zeta_{i}(t)$ are assumed to be Gaussian, with zero average, and correlations given by

$$
\left\langle\zeta_{i}(t) \zeta_{j}\left(t^{\prime}\right)\right\rangle=2 \delta_{i j} \delta\left(t-t^{\prime}\right), \quad i, j=1, \ldots, R .
$$

By performing a linearization of the function $\mathbf{f}(\mathbf{c}, \boldsymbol{\mu})$ around $\overline{\boldsymbol{\mu}}=\left\{\bar{\mu}_{1}, \ldots, \bar{\mu}_{R}\right\}$, Eq. (10) is replaced by

$$
\frac{d c_{i}}{d t}=f_{i}(\mathbf{c}, \overline{\boldsymbol{\mu}})+\sum_{j=1}^{R} g_{i j}(\mathbf{c}, \overline{\boldsymbol{\mu}}) \xi_{j}(t), \quad i=1, \ldots, N
$$

with

$$
g_{i j}(\mathbf{c}, \overline{\boldsymbol{\mu}})=\left.\frac{\partial f_{i}}{\partial \mu_{j}}\right|_{\boldsymbol{\mu}=\overline{\boldsymbol{\mu}}} .
$$

Eq. (9) represents a stochastic differential equation driven by multiplicative Ornstein-Uhlenbeck noise. For the sake of simplicity we make the further approximation that the noise correlation times $\tau_{i}$ 's be much smaller than any other time scale in the system. In this case the noises can be considered as virtually white. The process originally defined by (11), (3), and (4) becomes then to the first order in $\varepsilon_{i}$ and zero order in $\tau_{i}$ :

$$
\frac{d c_{i}}{d t}=f_{i}(\mathbf{c}, \overline{\boldsymbol{\mu}})+\sum_{j=1}^{R} \varepsilon_{j}^{1 / 2} g_{i j}(\mathbf{c}, \overline{\boldsymbol{\mu}}) \xi_{j}(t), \quad i=1, \ldots, N
$$

with

$$
\left\langle\xi_{i}(t) \xi_{j}\left(t^{\prime}\right)\right\rangle=2 \delta_{i j} \delta\left(t-t^{\prime}\right), \quad i, j=1, \ldots, R .
$$

Assuming the noise as virtually white is legitimate in all those cases when fluctuations are rapid, so that the zero $\tau_{i}$ expansion is justified. This may be the case in many different experimental situations, as it is highlighted for instance in [18]. Also enzyme activities fluctuations as related to conformational changes have been proven recently to have fast components [10, 17]. Environmental fluctuations, such as light fluctuations, can be experimentally realized so as to be very rapid as well. On the contrary, if fluctuations are not faster than the other typical processes of the system, the present scheme breaks down. In this case higher order terms in $\tau_{i}$ should be included to capture the full dynamics, as it is in any approximation scheme. However, the noise properties that we aim at discussing in this paper emerge already at the zeroth order in $\tau_{i}$. Our scope is to design a minimal model that accounts for the non-trivial properties of extrinsic noise.

As it is well known, the stochastic integral associated to the multiplicative noise term in (9) is not uniquely defined when white noise is assumed [12], and its evaluation needs to be supplemented with a prescription on how to define the discretized form of (9). The assumption that the white noise (10) is the limit of a correlated noise when the correlation time goes to zero resolves the ambiguity, and leads naturally to the adoption of the so-called Stratonovich prescription [12]. The same prescription is also valid when an elimination of fast variables is implemented, as in the reduction to the Michaelis-Menten form, as long as the equilibration time of the fast variables is anyway smaller than the correlation time of the noise [21].

A useful strategy to solve (9) is to derive the corresponding Fokker-Planck equation [31], and then compute the stationary probability density function. The Stratonovich form of the Fokker-Planck equation for the stochastic process (9) with the correlation (10) is

$$
\frac{\partial p(\mathbf{c}, t)}{\partial t}=-\sum_{i} \frac{\partial}{\partial c_{i}}\left\{\left[f_{i}(\mathbf{c}, \overline{\boldsymbol{\mu}})+G_{i}(\mathbf{c}, \overline{\boldsymbol{\mu}})\right] p(\mathbf{c}, t)\right\}+\sum_{i, j, k} \frac{\partial^{2}}{\partial c_{i} \partial c_{j}}\left\{\left[\varepsilon_{k} g_{i k}(\mathbf{c}, \overline{\boldsymbol{\mu}}) g_{j k}(\mathbf{c}, \overline{\boldsymbol{\mu}})\right] p(\mathbf{c}, t)\right\},
$$


where $p(\mathbf{c}, t)$ is a short notation for $p\left(\mathbf{c}, t \mid \mathbf{c}_{\mathbf{0}}, t_{0}\right)$, and

$$
G_{i}(\mathbf{c}, \overline{\boldsymbol{\mu}})=\sum_{j=1}^{R} \sum_{k=1}^{N} \varepsilon_{j} \frac{\partial g_{i j}(\mathbf{c}, \overline{\boldsymbol{\mu}})}{\partial c_{k}} g_{k j}(\mathbf{c}, \overline{\boldsymbol{\mu}})=\sum_{j=1}^{R} \sum_{k=1}^{N} \varepsilon_{j}\left\{\frac{\partial}{\partial c_{k}}\left[\frac{\partial}{\partial \mu_{j}} \sum_{l=1}^{M} S_{i l} v_{l}\right]\right\}\left\{\frac{\partial}{\partial \mu_{j}} \sum_{h=1}^{M} S_{k h} v_{h}\right\}
$$

is known as the Stratonovich drift, here specified for the kinetics (2).

Under the assumption of natural boundaries [12], the Stratonovich stationary probability distribution can be evaluated to be:

$$
p_{s}(\mathbf{c})=\exp \left\{\int^{\mathbf{c}} \sum_{i} d c_{i} \sum_{k} D_{i k}^{-1}(\mathbf{c}, \overline{\boldsymbol{\mu}})\left[\tilde{f}_{k}(\mathbf{c}, \overline{\boldsymbol{\mu}})-\sum_{j} \frac{\partial}{\partial c_{j}} D_{k j}(\mathbf{c}, \overline{\boldsymbol{\mu}})\right]\right\}
$$

where

$$
D_{i j}(\mathbf{c}, \overline{\boldsymbol{\mu}})=\sum_{k} \varepsilon_{k} g_{i k}(\mathbf{c}, \overline{\boldsymbol{\mu}}) g_{j k}(\mathbf{c}, \overline{\boldsymbol{\mu}}) \quad \text { and } \quad \tilde{f}_{i}(\mathbf{c}, \overline{\boldsymbol{\mu}})=f_{i}(\mathbf{c}, \overline{\boldsymbol{\mu}})+G_{i}(\mathbf{c}, \overline{\boldsymbol{\mu}}) .
$$

The maxima of the stationary probability distribution $p_{s}(\mathbf{c})$ are assumed to correspond to the macroscopic stable steady states of the pathway when noise is present, as they represent the states where the pathway is found for most of the time. Other indicators, such as the first moment, do not necessarily reflect well the behaviour of the system [22]. For this reason we shall focus on calculating the most probable states of the pathway, under the assumption that they represent the proper continuation of the deterministic states when noise is switched on. By differentiating eq. (13) the extrema $\mathbf{c}_{m}$ of the probability distribution are given by the expression:

$$
\mathbf{f}\left(\mathbf{c}_{m}, \overline{\boldsymbol{\mu}}\right)-\mathbf{G}\left(\mathbf{c}_{m}, \overline{\boldsymbol{\mu}}\right)=0 .
$$

The original fluctuations on parameters do not simply appear on the concentrations as mere fluctuations around their zero-noise values. Rather their effect is that of first shifting the value of the steady state concentrations, and then superimposing fluctuations upon them. This mechanism originates directly from the assumption of the noise being external. The small correlation present (in any case smaller than the other typical time scales of the system) makes the noise develop correlations with the dynamical variables at the same time [7]. As a consequence the multiplicative noise term in eq. (9) develops a non-zero average. The Stratonovich prescription is a way of extracting the non-zero average contribution in the limit of $\tau_{i}$ zero, and the corresponding modification of the dynamics. This modification is reflected in the change of the probability distribution with respect to the deterministic case, as well as of its zeroes, as shown by the condition (15). In general this accounts for a change in position, number, and stability properties of the steady states with respect to the deterministic solution.

\section{IMPLICATIONS FOR METABOLIC CONTROL ANALYSIS}

\section{3a. Standard Metabolic Control Analysis}

Metabolic Control Analysis (MCA) is a powerfull framework to relate local variables, such as enzymes activities or rate constants, to systemic variables, such as fluxes or concentrations. It focuses on establishing how the first exert control on the latter.

Ideally, MCA is based on a simple sensitivity analysis, which can be schematized as follows: $i$ ) Let the system relax to its steady state; ii) Apply a small perturbation; iii) Wait for relaxation onto the new steady state; iv) Measure the change in global variables (fluxes, concentrations, or other systemic variables).

The global effect of local changes is well described by a set of control coefficients, such as flux control coefficients,

$$
C_{v_{j}}^{J_{i}}=\frac{v_{j}}{J_{i}} \frac{\partial J_{i}}{\partial v_{j}}=\frac{\partial \ln J_{i}}{\partial \ln v_{j}}
$$

and concentration control coefficients,

$$
C_{v_{j}}^{c_{i}}=\frac{v_{j}}{c_{i}} \frac{\partial c_{i}}{\partial v_{j}}=\frac{\partial \ln c_{i}}{\partial \ln v_{j}}
$$


These can be derived in the approximation of small perturbations from the kinetic description of the metabolic network, for any given pathway, either branched or unbranched [15]. Notice that the form of control coefficients presented here, eqs. (16) and (17), contains the normalization factors $v_{j} / J_{i}$ and $v_{j} / c_{i}$. This form allows the characterizations of fractional changes and is suitable for the cases when none of the $J_{i} \mathrm{~s}$ or $c_{i} \mathrm{~s}$ is zero, which might happen in principle for certain combinations of the parameters. In that case unnormalized control coefficients may be used [15].

Control coefficients describe well the properties of the system as a whole. This particular fact is expressed through the so-called Summation Theorems, which relate coefficients for both concentrations and fluxes to each other:

$$
\sum_{j=1}^{M} C_{v_{j}}^{c_{i}}=0
$$

and

$$
\sum_{j=1}^{M} C_{v_{j}}^{J_{i}}=1
$$

Equation (18) states that for any metabolite in the network the sum of the concentration control coefficients of all rates equals zero. Similarly, for any flux the sum of all flux control coefficients equals 1, as implied by eq. (19). The fact that these sums are constrained demonstrates that control is shared among all rates in the pathway, and highlights the global properties of the network.

Local properties are equally important. In particular the response of individual rates to the perturbation of both internal and external variables may be very informative. This sensitivity is expressed in terms of so-called elasticity coefficients, whose definition is very similar to that of control coefficients:

$$
\pi_{z}^{v_{i}}=\frac{z}{v_{i}} \frac{\partial v_{i}}{\partial z}=\frac{\partial \ln v_{i}}{\partial \ln z}
$$

Here $z$ can be a dynamical variable, such as an internal metabolic concentration, or an external parameter, such as a reaction constant or a fixed external concentration. When $z$ is an internal metabolic concentration, Connectivity Theorems for both fluxes and concentrations can be derived [20]:

$$
\begin{aligned}
& \sum_{l=1}^{M} C_{v_{l}}^{J_{i}} \pi_{c_{j}}^{v_{l}}=0 \\
& \sum_{l=1}^{M} C_{v_{l}}^{c_{i}} \pi_{c_{j}}^{v_{l}}=-\delta_{i j} .
\end{aligned}
$$

Here $\delta_{i j}$ is the Kronecker symbol, equal to 1 for $i=j$, and 0 otherwise. Together with Summation Theorems, Connectivity Theorems allow for the calculation of the control coefficients from the knowledge of the elasticities, and therefore integrate between a local and a global description of the network.

In the present context we are also interested in the case when $z$ is a parameter, such as any of the $\mu_{j}$ s introduced in the previous section. Perturbation of parameters is described at the systemic level in terms of response coefficients, such as concentration response coefficients,

$$
R_{\mu_{j}}^{c_{i}}=\frac{\mu_{j}}{c_{i}} \frac{\partial c_{i}}{\partial \mu_{j}}=\frac{\partial \ln c_{i}}{\partial \ln \mu_{j}}
$$

and flux response coefficients,

$$
R_{\mu_{j}}^{J_{i}}=\frac{\mu_{j}}{J_{i}} \frac{\partial J_{i}}{\partial \mu_{j}}=\frac{\partial \ln J_{i}}{\partial \ln \mu_{j}}
$$

The interplay among response coefficients, control coefficients, and elasticities is formalized by the Partitioned Response relations, for both concentrations and fluxes,

$$
R_{\mu_{j}}^{c_{i}}=\sum_{i=1}^{M} C_{v_{i}}^{c_{i}} \pi_{\mu_{j}}^{v_{i}} \quad \text { and } \quad R_{\mu_{j}}^{J_{i}}=\sum_{i=1}^{M} C_{v_{i}}^{J_{i}} \pi_{\mu_{j}}^{v_{i}} .
$$


Eqs. (25) show that the system response originates locally through the sensitivity of local rates to the external perturbation, and is transfered to the systemic level through the mediation of the control that each rate has on the system variable of interest. Response coefficients are therefore determined by both local sensitivities to external effectors, and control exerted by individual rates. The set of control coefficients, response coefficients and elasticities, obeying Summation and Connectivity Theorems and/or Partitioned Response, represents a useful framework for connecting local and global properties of metabolic networks. In the next section we shall see that this mathematical structure is robust to extrinsic noise, but at the same time is sensitive to it through the acquired explicit dependency of all coefficients on the noise intensity.

\section{3b. Metabolic Control Analysis with extrinsic stochasticity}

One way to derive control, response, and elasticity coefficients, and their structural relationships, is to use the perturbative procedure mentioned in the previous section, and work out the corresponding linear approximation for small perturbations [16]. However, there is another elegant and compact way of deriving such properties when concentrations and rates are homogeneous functions.

Say that $f$ is a function of $n$ variables $x_{1}, \ldots x_{n}$, which satisfies a scaling relationship, such as:

$$
f\left(\lambda^{\alpha_{1}} x_{1}, \ldots, \lambda^{\alpha_{n}} x_{n}\right)=\lambda^{\gamma} f\left(x_{1}, \ldots, x_{n}\right) .
$$

Then it is possible to prove that the following equality holds true:

$$
\sum_{i=1}^{n} \alpha_{i} \frac{\partial \ln f}{\partial \ln x_{i}}=\gamma
$$

and $f$ is said to be a homogeneous function of degree $\gamma$ (Euler's Theorem). Homogeneity of concentrations and rates, of degree 0 and 1 respectively, has been shown to lead to Summation and Connectivity Theorems 13, 14].

In order to follow this approach, let us consider the equation that determines the maximum probability concentrations, eq. (15):

$$
\sum_{l=1}^{M} S_{i l} v_{l}-\sum_{j=1}^{R} \sum_{k=1}^{N} \sum_{l=1}^{M} \sum_{h=1}^{M} \varepsilon_{j}\left[\frac{\partial}{\partial c_{k}}\left(\frac{\partial v_{l}}{\partial \mu_{j}}\right)\right]\left[\frac{\partial v_{h}}{\partial \mu_{j}}\right] S_{i l} S_{k h}=0, \quad i=1, \ldots, N .
$$

It is not restrictive to assume specificity of the parameters with respect to the rates, namely

$$
\frac{\partial v_{l}}{\partial \mu_{j}} \neq 0 \quad \Rightarrow \quad \frac{\partial v_{h}}{\partial \mu_{j}}=0 \quad \forall h \neq l .
$$

This allows us to get rid of the sum over $h$ in (28). Also, by defining $R_{l}$ as the number of parameters entering in the kinetics of $v_{l}$, with $R=R_{1}+R_{2}+\ldots+R_{M}$, eq. (28) can be rewritten in the more compact form

$$
\sum_{l=1}^{M} S_{i l} \tilde{v}_{l}=0, \quad i=1, \ldots, N
$$

where

$$
\tilde{v}_{l}=v_{l}-v_{l}^{\varepsilon}, \quad l=1, \ldots, M
$$

and

$$
v_{l}^{\varepsilon}=\sum_{j=1}^{R_{l}} \sum_{k=1}^{N} \varepsilon_{j}\left[\frac{\partial}{\partial c_{k}}\left(\frac{\partial v_{l}}{\partial \mu_{j}}\right)\right]\left[\frac{\partial v_{l}}{\partial \mu_{j}}\right] S_{k l}, \quad l=1, \ldots, M .
$$

Equation (30) allows for a particularly transparent interpretation. Every rate present in the deterministic system $v_{l}$ appears together with the Stratonovich drift $v_{l}^{\varepsilon}$, made up of as many terms as the number $R_{l}$ of parameters affected by noise that enter the definition of $v_{l}$. Notice that in the case when the dependency on concentrations and parameters affected by noise is linear, the drifts (32) have the same analytical form as the zero noise rates $v_{l}$. In this case the 
noise simply causes a renormalization of parameter values. In contrast, for general nonlinear dependencies, eq. (32) defines interaction terms not necessarily of the same form as those defining the zero noise system.

Requiring that concentrations be homogeneous functions of degree 0 is equivalent to require the invariance of (30) under rescaling of the rates. In turn this rescaling needs to be defined consistently so that the rates themselves be homogeneoeus of degree 1 . In this way, while individual rates undergo a scale tansformation, concentrations will stay invariant.

It is clear that the scaling properties of the Stratonovich drift, eq. (32), depend on the specific form of the deterministic rates, and on the parameter that has been perturbed by noise. We shall focus on three different cases, as representative of most situations in metabolic networks: i) rates obeying the law of mass action, ii) rates described by Michaelis-Menten kinetics, and iii) rates described by reversible Michaelis-Menten kinetics.

Consider first a rate, $v_{\text {lin }}$, having the form given by the law of mass action, and let $\mu_{\text {lin }}$ be one of its parameters affected by noise. Since $v_{\text {lin }}$ is linear in $\mu_{\text {lin }}$, the rescaling $\mu_{\text {lin }} \rightarrow \lambda \mu_{\text {lin }}$ implies $v_{\text {lin }} \rightarrow \lambda v_{\text {lin }}$. Then the associated Stratonovich drift $v_{\text {lin }}^{\varepsilon}$ can be made to scale as $v_{\text {lin }}$ only if $\varepsilon_{\mu} \rightarrow \lambda \varepsilon_{\mu}$ where $\varepsilon_{\mu}$ is the intensity of the noise acting upon $\mu_{\text {lin }}$. Therefore the rate $\tilde{v}_{\text {lin }}$ scales as $\lambda$, that is it is homogeneous of degree 1 , if

$$
v_{\text {lin }} \rightarrow \lambda v_{\text {lin }} \text { and } \quad \varepsilon_{\mu} \rightarrow \lambda \varepsilon_{\mu} .
$$

Next consider rates of the irreversible Michaelis-Menten form, $v_{\mathrm{MM}}=V_{\max } S /\left(K_{m}+S\right)$, with $S$ the substrate concentration, and $V_{\max }$ and $K_{m}$ maximal velocity and Michaelis-Menten constant respectively. The general form of the scaling properties of $v_{\mathrm{MM}}$ is

$$
v_{\mathrm{MM}}\left(\lambda^{\alpha} V_{\max }, \lambda^{0} K_{m}\right)=\lambda^{\alpha} v_{\mathrm{MM}}\left(V_{\max }, K_{m}\right) .
$$

This implies $v_{\mathrm{MM}} \rightarrow \lambda v_{\mathrm{MM}}$, when $V_{\max } \rightarrow \lambda V_{\max }$ and $K_{m} \rightarrow K_{m}$. To find the scale transformation of the associated Stratonovich drift $v_{\mathrm{MM}}^{\varepsilon}$, we consider separately the two cases, when the noise acts either upon $V_{\max }$, or upon $K_{m}$. If $V_{\max }$ is affected by a noise having intensity $\varepsilon_{V}$, the corresponding $v_{\mathrm{MM}}^{\varepsilon}$ scales as $v_{\mathrm{MM}}$ again if $\varepsilon_{V}$ scales as $\lambda$. So that the rate $\tilde{v}_{\text {MM }}$ scales as $\lambda$ if

$$
v_{\mathrm{MM}} \rightarrow \lambda v_{\mathrm{MM}} \text { and } \quad \varepsilon_{V} \rightarrow \lambda \varepsilon_{V}
$$

Notice that (35) is the same set of scale transformations as (33). This is not surprising since the dependency of any Michaelis-Menten type of rate on maximal velocities is in fact linear, and therefore the previous case must apply.

In contrast, if $K_{m}$ is the parameter of $v_{\mathrm{MM}}$ perturbed by a noise with intensity $\varepsilon_{K}$, then the corresponding $v_{\mathrm{MM}}^{\varepsilon}$ scales as $\lambda^{2} \varepsilon_{K}$ when $V_{\max } \rightarrow \lambda V_{\max }$ and $K_{m} \rightarrow K_{m}$. Therefore, imposing $\varepsilon_{K}$ to scale as $\lambda^{-1}$ makes $v_{\mathrm{MM}}^{\varepsilon}$ to scale as $\lambda$, which is what we want in order for $\tilde{v}_{\mathrm{MM}}$ to be homogeneous of degree 1 :

$$
v_{\mathrm{MM}} \rightarrow \lambda v_{\mathrm{MM}} \quad \text { and } \quad \varepsilon_{K} \rightarrow \lambda^{-1} \varepsilon_{K} .
$$

The same result can be obtained also in the case of the reversible Michaelis-Menten rate,

$$
v_{\mathrm{RMM}}=\frac{\frac{V_{\max }^{\mathrm{f}}}{K_{S}} S-\frac{V_{\max }^{\mathrm{b}} P}{K_{P}} P}{1+\frac{S}{K_{S}}+\frac{P}{K_{P}}},
$$

where $V_{\max }^{\mathrm{f}}$ and $V_{\max }^{\mathrm{b}}$ are maximal velocities for the forward and backward reactions respectively, and $K_{S}$ and $K_{P}$ are the Michaelis-Menten constants associated to the substrate $S$ and the product $P$. The scaling properties of $v_{\mathrm{RMM}}$ are similar to the irreversible case:

$$
v_{\mathrm{RMM}}\left(\lambda^{\alpha} V_{\max }^{\mathrm{f}}, \lambda^{\alpha} V_{\max }^{\mathrm{b}}, \lambda^{0} K_{S}, \lambda^{0} K_{P}\right)=\lambda^{\alpha} v_{\mathrm{RMM}}\left(V_{\max }^{\mathrm{f}}, V_{\max }^{\mathrm{b}}, K_{S}, K_{P}\right) .
$$

As before, if $V_{\max }^{\mathrm{f}}$ and/or $V_{\max }^{\mathrm{b}}$ are affected by noise, $\tilde{v}_{\mathrm{RMM}}$ scales as $\lambda$ if

$$
v_{\mathrm{RMM}} \rightarrow \lambda v_{\mathrm{RMM}} \quad \text { and } \quad \varepsilon_{V} \rightarrow \lambda \varepsilon_{V}
$$

On the other hand, if any of the Michaelis-Menten constants are affected by noise, then the scaling

$$
v_{\mathrm{RMM}} \rightarrow \lambda v_{\mathrm{RMM}} \quad \text { and } \quad \varepsilon_{K} \rightarrow \lambda^{-1} \varepsilon_{K} .
$$

makes sure that $\tilde{v}_{\mathrm{RMM}}$ is homogeneous of degree 1 . 
In summary, under rescaling $v_{l} \rightarrow \lambda v_{l}$, the rates $\tilde{v}_{l}$ are homogeneous functions of degree 1 if the noise intensities of any parameter appearing linearly in the rates are rescaled as $\lambda$ (including maximal velocities of possible MichaelisMenten rates), and the noise intensities associated to Michaelis-Menten constants are rescaled as $\lambda^{-1}$. Also, because of the invariance of (30) under this same rescaling, the maximum probability concentrations are homogeneous functions of degree 0. For other possible types of parameter dependencies, the proper scale transformation need to be sought case by case by applying similar arguments.

By using Euler's Theorem, the homogeneity of concentrations and rates leads directly to the Summation Theorems for the respective control coefficients. In particular, from the scaling relation

$$
\boldsymbol{c}^{m}\left(\lambda \tilde{v}_{1}, \ldots, \lambda \tilde{v}_{M}\right)=\boldsymbol{c}^{m}\left(\tilde{v}_{1}, \ldots, \tilde{v}_{M}\right),
$$

and by using (27), we readily obtain

$$
\sum_{j=1}^{M} \frac{\partial \ln c_{i}^{m}}{\partial \ln \tilde{v}_{j}}=0
$$

that is

$$
\sum_{j=1}^{M} C_{\tilde{v}_{j}}^{c^{m}}=0
$$

Eq. (43) proves that the Summation Theorem for concentration control coefficients is still valid when stochasticity is present in the form of noise on parameters.

Similarly, by defining the macrosopic fluxes as the fluxes evaluated in correspondence of the most probable concentrations, $\boldsymbol{J}^{m}=\tilde{\boldsymbol{v}}\left(\mathbf{c}^{m}\right)$, it is clear that

$$
\boldsymbol{J}^{m}\left(\lambda \tilde{v}_{1}, \ldots, \lambda \tilde{v}_{M}\right)=\lambda \boldsymbol{J}^{m}\left(\tilde{v}_{1}, \ldots, \tilde{v}_{M}\right) .
$$

Euler's Theorem may be invoked again, and implies

$$
\sum_{j=1}^{M} C_{\tilde{v}_{j}}^{J_{i}^{m}}=1
$$

which states that the Summation Theorem for flux control coefficients is valid as well, even in presence of extrinsic noise.

The substitution of the zero noise rates $v_{l}$ with the rates (31), which obey the same scaling properties, allows also to derive in a standard fashion Connectivity Theorems [14] and Partitioned Response [16]:

$$
\sum_{l=1}^{M} C_{\tilde{v}_{l}}^{J_{i}^{m}} \pi_{c_{j}^{m}}^{\tilde{v}_{l}}=0 \quad \text { and } \quad \sum_{l=1}^{M} C_{\tilde{v}_{l}}^{c_{i}^{m}} \pi_{c_{j}^{m}}^{\tilde{v}_{l}}=-\delta_{i j} .
$$

and

$$
R_{\mu_{j}}^{c_{i}}=\sum_{i=1}^{M} C_{\tilde{v}_{i}}^{c_{i}^{m}} \pi_{\mu_{j}}^{\tilde{v}_{i}} \quad \text { and } \quad R_{\mu_{j}}^{J_{i}^{m}}=\sum_{i=1}^{M} C_{\tilde{v}_{i}}^{J_{i}} \pi_{\mu_{j}}^{\tilde{v}_{i}} .
$$

It is interesting to note that new elasticity coefficients can be defined, which describes the local effect of perturbations of the noise intensities,

$$
\pi_{\varepsilon_{j}}^{\tilde{v}_{i}}=\frac{\varepsilon_{j}}{\tilde{v}_{i}} \frac{\partial \tilde{v}_{i}}{\partial \varepsilon_{j}}
$$

together with new response coefficients, for both concentrations and fluxes, which in turn describe the effect of the noise on the sytemic variables:

$$
R_{\varepsilon_{j}}^{c_{i}^{m}}=\frac{\varepsilon_{j}}{c_{i}^{m}} \frac{\partial c_{i}^{m}}{\partial \varepsilon_{j}} \quad \text { and } \quad R_{\varepsilon_{j}}^{J_{i}^{m}}=\frac{\varepsilon_{j}}{J_{i}^{m}} \frac{\partial J_{i}^{m}}{\partial \varepsilon_{j}}
$$




$$
\mathrm{A} \underset{k_{-1}}{\stackrel{k_{1}}{\rightleftarrows}} \mathrm{X} \underset{k_{-2}}{\stackrel{k_{2}}{\rightleftarrows}} \mathrm{B}
$$

FIG. 1: The elementary reaction pathway chosen as an illustration of the effect of extrinsic fluctuations.

Summation Theorems (43) and (45), Connectivity Theorems (46), and Partitioned Response (47) show that the mathematical structure of the network is robust against extrinsic noise. This property is non trivial, because it might be expected that extrinsic noise might break the homogeneity of the system. Partitioned Response originates in fact from the breakdown of homogeneity of fluxes, but in the same way as in the deterministic case. The fact that the Stratonovich drift obeys the same scale transformation as the zero noise rates guarantees that these properties are preserved. However, it should be noted that individual control coefficients, as well as response coefficients and elasticities, depend explicitly on the noise intensity. This feature demonstrates that extrinsic noise can in fact act as a control mechanism. It modifies the local rates, as formalized by the corresponding elasticy coefficients, and propagates up to the systemic level to affect global variables.

\section{AN EXPLICIT EXAMPLE}

As an example of the theory presented, let us consider the chemical reaction depicted in Fig. 11 Despite its simplicity it serves well the purpose of illustrating the notion of control by noise.

Let us define the concentrations of the species $A, B$, and $X$ as $a, b$, and $x$ respectively. Let us assume that the constant $k_{2}$ is the only parameter undergoing stochastic fluctuations. This leads to the following Stratonovich SDE

$$
\frac{d x}{d t}=v_{1}-v_{2}-\varepsilon^{1 / 2} x \xi(t)
$$

where $\xi(t)$ is Gaussian white noise. The deterministic reactions $v_{1}$ and $v_{2}$ are given in terms of the rate constants defined in Fig. 11a as

$$
\begin{aligned}
& v_{1}=k_{1} a-k_{-1} x, \\
& v_{2}=k_{2} x-k_{-2} b .
\end{aligned}
$$

Notice that Equation (50) is exact at all orders in $\varepsilon$, because of the linear dependence of the kinetics on $k_{2}$. In general, this dependence may be nonlinear, and the equation corresponding to (50) in the nonlinear case should be considered valid only at the leading order in $\varepsilon$.

By following Section 2, we compute the stationary concentrations and fluxes in order to derive the corresponding control coefficients. In one dimension, with extrinsic fluctuations acting upon only one parameter, eq. (13) becomes:

$$
p_{s}(x)=\frac{\mathcal{N}}{g(x)} \exp \left(\frac{1}{\varepsilon} \int^{x} \frac{f\left(x^{\prime}\right)}{g^{2}\left(x^{\prime}\right)} d x^{\prime}\right),
$$

with $f(x)=v_{1}(x)-v_{2}(x), g(x)=-x$ and $\mathcal{N}$ a normalization constant that can be directly calculated by imposing $\int_{o}^{\infty} p_{s}(x) d x=1$. The explicit expression of $p_{s}(x)$ is then

$$
p_{s}(x)=\left(\frac{k_{1} a+k_{-2} b}{\varepsilon}\right)^{\left(k_{-1}+k_{2}\right) / \varepsilon} \frac{x^{-\left(1+\left(k_{-1}+k_{2}\right) / \varepsilon\right)}}{\Gamma\left(\left(k_{-1}+k_{2}\right) / \varepsilon\right)} \exp \left(-\frac{k_{1} a+k_{-2} b}{\varepsilon x}\right) .
$$

The extrema of $p_{s}(x)$, as from eq. (15), result in $v_{1}-v_{2}-\varepsilon x=0$, which gives

$$
x_{m}=\frac{k_{1} a+k_{-2} b}{k_{-1}+k_{2}+\varepsilon} .
$$

Notice that all the moments of the distribution (54) can be calculated, and in particular we have:

$$
\langle x\rangle=\int_{0}^{\infty} d x x p_{s}(x)=\frac{k_{1} a+k_{-2} b}{k_{-1}+k_{2}-\varepsilon}, \quad \varepsilon<k_{-1}+k_{2} .
$$



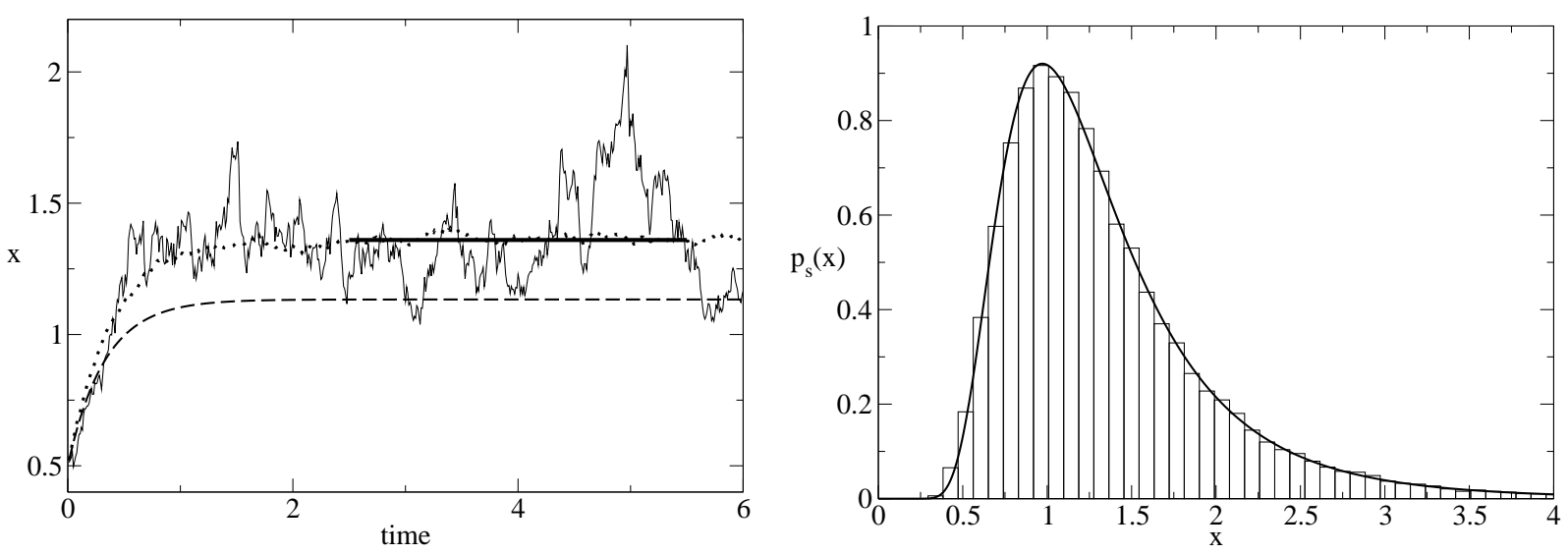

FIG. 2: Left panel: Dependency over time of the concentration $x$, as given by eq. (50). Parameters have been set arbitrarily to the following values: $k_{1}=1 \mathrm{~min}^{-1}, k_{-1}=1 \mathrm{~min}^{-1}, k_{2}=2 \mathrm{~min}^{-1}, k_{-2}=1.2 \mathrm{~min}^{-1}$. External concentrations have been taken as $a=1 \mathrm{mM}$ and $b=2 \mathrm{mM}$, while the noise intensity has been fixed to $\varepsilon=0.5$. The dashed curve corresponds to the solution of the deterministic equation, the spiky and dotted lines show the value of the concentration averaged respectively over 10 and 1000 realizations of the noise. The thick straight line is the value of the average concentration has from eq. (56), namely $\langle x\rangle=1.36 \mathrm{mM}$ with the chosen parameters. Right panel: The stationary probability distribution (54) (solid line), versus the estimate coming from the numerical solution of eq. (50), with the parameters as above. The maximum is at $x_{m}=0.97 \mathrm{mM}$, whereas the deterministic value is $x_{m}=1.13 \mathrm{mM}$.

Fig. 2 shows a direct numerical simulation of the SDE (50) with the Stratonovich prescription. The numerical integration scheme is standard (see for instance 11]). In the left panel of Fig. 2 comparison is shown between the deterministic solution, namely the solution of (50) with the noise term switched off, the full stochastic solution, and the expectation $\langle x\rangle$, averaged over 10 and 1000 realizations of the noise. The theoretical value of $\langle x\rangle$ as given by (56) is also plotted for comparison. In the right panel of the same figure we show the probability density (54) and its numerical evaluation from the solution of eq. (50). Notice that the maximum of the probability density has undergone indeed a shift with respect to the deterministic value, by changing its value from $x_{m}=1.13 \mathrm{mM}$ (deterministic) to $x_{m}=0.97 \mathrm{mM}$ (stochastic). The same is true for the expectation $\langle x\rangle$, whose value has changed from $\langle x\rangle=1.13 \mathrm{mM}$ (deterministic) to $\langle x\rangle=1.36 \mathrm{mM}$ (stochastic).

In summary, the effect caused by extrinsic fluctuations affecting the parameter $k_{2}$ is a shift in the value of the maximum of the probability density, which induces a shift also on the value of its first moment. Notice that this mechanism corresponds effectively to resetting the value of the parameter $k_{2}$ to $k_{2}+\varepsilon$. This resetting is non-trivial in that it rests on the action of the fluctuations only, which shift the value of $k_{2}$ effectively perceived by the system. Nonetheless the bare $k_{2}$ is blocked to its zero-noise value. Due to the linearity of the system, this is the only effect, and more subtle modifications, such as in the number of stationary states of the probability distribution, or in their stability properties, are not present.

The same resetting appears in the expression for the flux as well, which becomes thereby explicitly dependent on the noise intensity. In fact, the rates (31) read in this case:

$$
\begin{aligned}
& \tilde{v}_{1}=k_{1} a-k_{-1} x, \\
& \tilde{v}_{2}=\left(k_{2}+\varepsilon\right) x-k_{-2} b,
\end{aligned}
$$

and their direct evaluation at the value $x_{m}$ results in:

$$
J^{m}=J_{1}^{m}=J_{2}^{m}=\tilde{v}_{1}\left(x_{m}\right)=\tilde{v}_{2}\left(x_{m}\right)=\frac{k_{1}\left(k_{2}+\varepsilon\right) a-k_{-1} k_{-2} b}{k_{-1}+k_{2}+\varepsilon} .
$$

The noise dependency of the maximum probability concentration (55) and of the corresponding flux (59) can be interpreted within Metabolic Control Analysis. To this aim, we compute now control coefficients, response coefficients and elasticies, and verify that the Summation and Connectivity Theorems are fulfilled, as from Section 3b.

The concentration control coefficients result in

$$
C_{\tilde{v}_{1}}^{x}=\frac{k_{1}\left(k_{2}+\varepsilon\right) a-k_{-1} k_{-2} b}{\left(k_{-1}+k_{2}+\varepsilon\right)\left(k_{1} a+k_{-2} b\right)},
$$



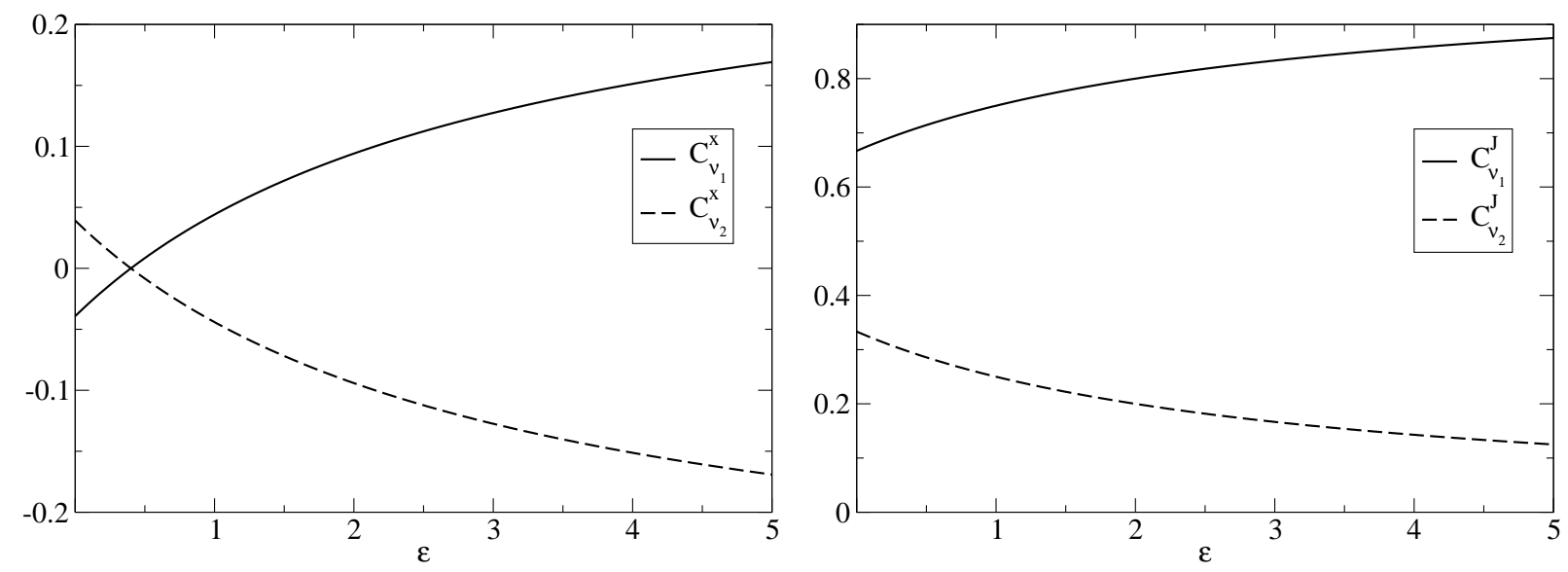

FIG. 3: Concentration (left panel) and flux (right panel) control coefficients as from eqs. (60)-(63) as functions of $\varepsilon$. Parameters setting is as in the caption of Fig. 2

$$
C_{\tilde{v}_{2}}^{x}=-\frac{k_{1}\left(k_{2}+\varepsilon\right) a-k_{-1} k_{-2} b}{\left(k_{-1}+k_{2}+\varepsilon\right)\left(k_{1} a+k_{-2} b\right)}
$$

and similarly for the flux $J^{m}$,

$$
\begin{aligned}
C_{\tilde{v}_{1}}^{J} & =\frac{k_{2}+\varepsilon}{k_{-1}+k_{2}+\varepsilon}, \\
C_{\tilde{v}_{2}}^{J} & =\frac{k_{-1}}{k_{-1}+k_{2}+\varepsilon} .
\end{aligned}
$$

It is immediate to verify that the Summation Theorems hold true, as expected:

$$
C_{v_{1}}^{x}+C_{v_{2}}^{x}=0 \quad \text { and } \quad C_{v_{1}}^{J}+C_{v_{2}}^{J}=1 .
$$

In Fig. 3 we present a numerical evaluation of expressions (60)-(63) as functions of the noise intensity $\varepsilon$, with parameters chosen as indicated in the caption of Fig. 2. As eqs. (55) and (59) show, the effect of the noise is to allow an extra tuning mechanism for modulating the systemic variables. Futhermore, noise may also allow for a change of the control that individual rates exert on the whole pathway. In other words, the dependency of the control coefficients on the noise makes it possible for the system to rebalance controls, and possibly to explore dynamical regions that might not be accessible given the standard parameter values. Already in this simple linear system, this effect can be so dramatic as to invert the sign of the control of the two rates on the concentration, as the left panel of Fig. 3 shows. Of course this extra tuning is always constrained by the respective Summation Theorems.

As proven in section $3 \mathrm{~b}$, Connectivity Theorems are also preserved. It is a simple matter to compute the elasticities,

$$
\begin{aligned}
& \pi_{x}^{\tilde{v}_{1}}=-\frac{k_{1} k_{-1} a+k_{-1} k_{-2} b}{k_{1}\left(k_{2}+\varepsilon\right) a-k_{-1} k_{-2} b}, \\
& \pi_{x}^{\tilde{v}_{2}}=\frac{k_{1}\left(k_{2}+\varepsilon\right) a+k_{-2}\left(k_{2}+\varepsilon\right) b}{k_{1}\left(k_{2}+\varepsilon\right) a-k_{-1} k_{-2} b},
\end{aligned}
$$

and verify that

$$
C_{\tilde{v}_{1}}^{x} \pi_{x}^{\tilde{v}_{1}}+C_{\tilde{v}_{2}}^{x} \pi_{x}^{\tilde{v}_{2}}=-1 \quad \text { and } \quad C_{\tilde{v}_{1}}^{J} \pi_{x}^{\tilde{v}_{1}}+C_{\tilde{v}_{2}}^{J} \pi_{x}^{\tilde{v}_{2}}=0 .
$$

On the other hand, elasticities and response coefficients with respect to noise turn out to be

$$
\begin{aligned}
\pi_{\varepsilon}^{\tilde{v}_{1}} & =0, \\
\pi_{\varepsilon}^{\tilde{v}_{2}} & =\frac{\varepsilon\left(k_{1} a+k_{-2} b\right)}{k_{1}\left(k_{2}+\varepsilon\right) a-k_{-1} k_{-2} b},
\end{aligned}
$$


and

$$
\begin{aligned}
& R_{\varepsilon}^{x}=-\frac{\varepsilon}{k_{-1}+k_{2}+\varepsilon}, \\
& R_{\varepsilon}^{J}=\frac{\varepsilon k_{-1}\left(k_{1} a+k_{-2} b\right)}{\left(k_{-1}+k_{2}+\varepsilon\right)\left(k_{1}\left(k_{2}+\varepsilon\right) a-k_{-1} k_{-2} b\right)},
\end{aligned}
$$

and obey the Partitioned Response:

$$
R_{\varepsilon}^{x}=C_{\tilde{v}_{1}}^{x} \pi_{\varepsilon}^{\tilde{v}_{1}}+C_{\tilde{v}_{2}}^{x} \pi_{\varepsilon}^{\tilde{v}_{2}}=C_{\tilde{v}_{2}}^{x} \pi_{\varepsilon}^{\tilde{v}_{2}} \quad \text { and } \quad R_{\varepsilon}^{J}=C_{\tilde{v}_{1}}^{J} \pi_{\varepsilon}^{\tilde{v}_{1}}+C_{\tilde{v}_{2}}^{J} \pi_{\varepsilon}^{\tilde{v}_{2}}=C_{\tilde{v}_{2}}^{J} \pi_{\varepsilon}^{\tilde{v}_{2}} .
$$

The elasticity (70) highlights the local effect of noise, and the response coefficients (71) and (72) for concentrations and fluxes are a manifestation of its global effect at the systemic level. While the action of noise on control coefficients is only a modification of the existiting ones, which become noise dependent, the elasticity (70) and the response coefficients (711) and (72) are truly "new" coefficients, which are turned on by the presence of noise. Nonetheless they obey Partitioned Response in the standard fashion. In this sense, the mathematical structure of the theory is generally preserved.

\section{CONCLUSION AND OUTLOOK}

Metabolic Control Analysis is a fundamental framework that links both conceptually and practically local and global properties of metabolic networks. In this paper we propose a way of generalizing MCA, and its predictive power at the systemic level, when fluctuations are present in control parameters, and the kinetics are affected by so-called extrinsic noise.

The proposed generalization relies on the acquired explicit dependency of all standard MCA coefficients on noise intensity, and on the definition of new elasticity and response coefficients accounting for noise perturbations. Even though Summation and Connectivity Theorems, as well as Partitioned Response, are shown to be robust against noise, noise introduces an extra tuning mechanism, capable of modifying the local and global properties of the pathway.

In fact, through the noise dependency of the MCA coefficients, we aim at putting forward a new way of looking at noise in metabolic networks. We propose to go beyond the interpretation of noise as a mere experimental nuisance, and to explore its potentiality to exert external functional control onto the system. As a result of our analysis, the behaviour of the pathway can be regulated by the insertion of ad hoc random fluctuations on properly chosen parameters.

Intrinsic noise, related for instance to the low copy number of molecules or to protein conformational fluctuations, can also be described by this approach. From within a modelling perspective, deciding whether noise is internal or external to the system is so delicate an issue as defining the pathway of interest itself. In an integrated description of the full network all noise has an internal origin. However, the description of reduced modules, as opposed to the fully integrated approach, requires the adoption of effective kinetics, where intrinsic noise is translated into extrinsic noise on parameters. The predictions of these effective kinetics are the ones, which should be compared with experimental data, or to full stochastic simulations.

This is particularly relevant when the system is characterized by a time-scale separation between slow and fast variables. If the latter are eliminated by standard techiques [9], their dynamical contribution is rewritten in parametric form. Accordingly original intrinsic fluctuations acting on fast variables are perceived as extrinsic noise in the reduced system. As long as the reduction procedure is carried out before the approximation of white noise [21], the reduced dynamics is modified by the Stratonovich drift. This scenario is confirmed for instance in [9], where an improved version of the Linear Noise Approximation combined with the elimination of fast variables in a simple metabolic network shows a very good agreement with the results of a direct stochastic simulation [9].

In all other cases intrinsic noise can be described by standard tools such as Master Equations, and possibly their Fokker-Planck [31] or Linear Noise approximations [9]. Effective Langevin descriptions can then be derived, but, apart from some exceptions [7], these are generally characterized by zero average multiplicative noise terms, with no Stratonovich drift associated. This implies that no control mechanism can be expected.

A recent work by Levine and Hwa [23] confirms this prediction. The Authors of [23] study the effect that intrinsic noise, as associated to copy number fluctuations of metabolic species, may have on metabolic pathways. The conclusion of the Authors is that noise "does not propagate", being the fluctuations of the metabolites in the pathway uncorrelated from each other. This is fully consistent with our framework. However possible correlations among metabolic fluctuations might in fact arise when fluctuations of enzymes common to two or more metabolites are considered. Our treatment of extrinsic noise aims to address this particular case. 
In this respect, the possible internal origin of extrinsic noise suggests an even more interesting role of stochastic fluctuations. Robustness of biochemical systems to perturbations is often thought to be a fundamental property, and selection of robust traits has been proposed as a driving principle of evolution [19]. Noise of course is no exception, and the capability of preserving functionality despite noise is certainly a fundamental feature of living systems (see for instance [8] for an example). A different perspective, however, emerges from considering cellular noise as a genetic response to chemical or physical constraints, as represented for instance by the values of reaction constants in a biochemical network. As we have shown, the operational point of the network is determined by the parameter values jointly with the intensity of the external noise acting on the system. However, as discussed above, external noise may have its origin in intrinsic noise, associated for instance to concentration fluctuations. It is a suggestive picture that biochemical networks would have evolved in such a way that concentration fluctuations allow the network to gain access to otherwise dynamically forbidden regions [32]. Noise then would appear as a result of positive selection on gene expression levels rather than as an external factor causing "defensive" architectural evolution. To what extent evolution can positively select for stochastic dynamics as opposed to deterministic ones is a fundamental and intriguing question.

The theory proposed in this paper applies to both linear and nonlinear dependencies of reaction rates on parameters and concentrations. In the linear case the analysis is particularly simple. The effect of the noise is perceived by the system as a renormalization of the relevant parameters.

When nonlinear dependencies are considered, may be that the effect of the noise cannot be reabsorbed as a renormalization of bare parameters only, and the overall dynamics is expected to be generally more complex. This case has been object of intense study, and the theory of noise-induced transition has emerged 22]. Because of the nonlinearities, the effect of the noise is not limited to a shift in the extrema of the probability distribution, to which our theory applies for small enough noise intensities, but new extrema may appear, and the system can jump from one to the other driven by random fluctuations. Even though this mechanism is well understood at the kinetic level, at the systemic level its description requires a proper MCA, which needs to be valid for large jumps between stationary states. This is the subject of some recent literature [1, 2], and the corresponding analysis when extrinsic noise is present will be the subject of a further study.

As a final remark, we would like to mention also the recent extensions of MCA that have been discussed in 27] to account for spatial dependencies. These are relevant for instance in signal transduction networks, where protein diffusion plays an important role in the signaling process [30]. Modelling these systems relies on defining proper Reaction-Diffusion dynamics, for which the standard MCA needs to be extended accordingly. Our paper follows conceptually the same spirit of [27], as it aims at identifying on general grounds all processes that may have relevant biological implications. The simultaneous treatment of both noise and spatial dependencies in stochastic non homogeneous systems is in turn a combination of the theory of [27] and the theory presented in this paper. This will also be the subject of a further study.

[1] Acerenza, L., and Ortega, F.: Metabolic Control Analysis for large changes: Extension to variable elasticity coefficients, IEE Proc. Sys. Biol. 153, 323-326 (2006).

[2] Acerenza L., and Ortega, F.: Modular Metabolic Control Analysis of large responses, FEBS Journal 274, 188-201 (2007).

[3] L. Alberghina and H.V. Westerhoff, Systems Biology: Definition and perspectives, Springer (2005).

[4] Aurell, E., and Sneppen, K.:Epigenetics as a first exit problem, Phys. Rev. Lett. 88, 048101-1-4 (2002).

[5] Becskei, A., Kaufmann, B.B., van Oudenaarden, A.: Contributions of low molecule number and chromosomal positioning to stochastic gene expression, Nature Genetics 37, 937-943 (2005).

[6] Blake, W.J., Kærn, M., Cantor, C.R., and Collins, J.J.: Noise in eukaryotic gene expression, Nature 422, 633-637 (2003).

[7] Carrillo, O., Ibañes, M., García-Ojalvo, J., Casademunt, J., Sancho, J.M.: Intrinsic noise-induced phase transitions: Beyond the noise interpretation, Phys. Rev. E 67, 046110-1-9 (2003).

[8] Eldar, A., Dorfman, R., Weiss, D., Ashe, H., Shilo, B.-Z., and Barkai, N.: Robustness of the BMP morphogen gradient in Drosophila embryonic patterning, Nature 419, 304-308 (2002).

[9] Elf, J., and Ehrenberg, M.: Fast Evaluation of Fluctuations in Biochemical Networks With the Linear Noise Approximation, Gen. Res. 13, 2475-2484 (2003).

[10] English, B.P., Min, W., van Oijen, A.M., Lee, K.T., Luo, G., Sun, H., Cherayil, B.J., Kou, S.C., Xie, X.S.: Ever-fluctuating single enzyme molecules: Michaelis-Menten equation revisited, Nature Chem. Bio. 287 (2006).

[11] García-Ojalvo J., and Sancho, J.M.: Noise in Spatially Extended Systems, Springer (1999).

[12] Gardiner, C.W.: Handbook of Stochastic Methods for Physics, Chemistry and the Natural Sciences, Springer (1997).

[13] Giersch, C.: Control analysis of metabolic networks. 1. Homogeneous functions and the summation theorems for control coefficients, Eur. J. Biochem. 174, 509-513 (1988). 
[14] Giersch, C.: Control analysis of biochemical Pathways: A novel procedure for calculating control coefficients, and an additional theorem for branched pathways, J. Theor. Biol. 134, 451-462 (1988).

[15] Heinrich, R., and Schuster, S.: The regulation of cellular systems, Chapman \& Hall (1996).

[16] Hofmeyr, J.-H.S.: Metabolic control analyis in a nutshell, Online Proceedings of the 2nd International Conference on Systems Biology, Caltech 2001.

[17] Ishikawa, H., Kwak, K., Chung, J.K., Kim, S., and Fayer, M.D.: Direct observation of fast protein conformational switching, PNAS 105 8619-8624 (2008).

[18] Kar, S, and Ray, D.S.: Collapse and Revival of Glycolytic Oscillation, Phys. Rev. Lett. 90, 238102-1-238102-4 (2003).

[19] Kitano, H.: Biological Robustness, Nature Rev. Gen. 5, 826-837 (2004).

[20] Klipp, E., Herwig, R., Kowald, A., and Wierling, C.: Systems Biology in Practice: Concepts, Implementation and Application, Wiley-VCH (2005).

[21] Kupferman, R., Pavliotis, G.A., and Stuart, A.M.: Itô versus Stratonovich white-noise limits for systems with inertia and colored multiplicative noise, Phys. Rev. E 70, 036120-1-9 (2004).

[22] Horsthemke W., and Lefever, R.: Noise-induced Transitions, Springer (1984).

[23] Levine, E., and Hwa, T.: Stochastic fluctuations in metabolic pathways, PNAS 104, 9224-9229 (2007).

[24] Ozbudak, E.M., Thattai, M., Kurtser, I., Grossman, A.D., and van Oudenaarden, A.: Regulation of noise in the expression of a single gene, Nature genetics 31, 69-73 (2002).

[25] Paulsson, J.: Summing up the noise in gene networks, Nature 427, 415-418 (2004).

[26] Pedraza, J.M., and van Oudenaarden, A.: Noise propagation in gene networks, Science 307, 1965-1969 (2005).

[27] Peletier, M.A., Westerhoff, H.V., and Kholodenko, B.N.: Control of spatially heterogeneous and time-varying cellular reaction networks: a new summation law, J. Theor. Biol. 225, 477-487 (2003).

[28] Swain, P.S., Elowitz, M.B., and Siggia, E.D.: Intrinsic and extrinsic contributions to stochasticity in gene expression, PNAS 99, 12795-12800 (2002).

[29] Tănase-Nicola, S., Warren, P.B., Rein ten Wolde, P.: Signal detection, modularity and the correlation between extrinsic and intrinsic noise in biochemical networks, Phys. Rev. Lett. 97, 068102-1-4 (2006).

[30] Takahashi, K., Arjunan, S.N.V., and Tomita, M.: Space in systems biology of signaling pathways - towards intracellular molecular crowding in silico, FEBS Lett. 579, 1783-1788 (2005).

[31] van Kampen, N.G.: Stochastic processes in physics and chemistry, Elsevir, Amsterdam (1997).

[32] Vilar, J.M.G., Kueh, H.Y., Barkai, N., Leibler, S.: Mechanisms of noise-resistance in genetic oscillators, PNAS 99, 5988$5992(2002)$.

[33] Zhou, T., Chen, L., and Aihara, K.: Molecular communication through stochastic synchronization induced by extracellular fluctuations, Phys. Rev. Lett. 95, 178103-1-178103-4 (2005). 